\title{
Computing the Characteristics of a SubSegment of a Digital Straight Line in Logarithmic Time
}

\author{
Mouhammad Said ${ }^{1,2}$ and Jacques-Olivier Lachaud ${ }^{1}$ \\ 1 Laboratoire de Mathématiques, UMR 5127 CNRS, Université de Savoie, \\ 73376 Le Bourget du Lac, France \\ \{mouhammad.said, jacques-olivier.lachaud\}@univ-savoie.fr \\ 2 LAIC, Univ. Clermont-Ferrand, \\ IUT, Campus des Cézeaux, 63172 Aubière Cedex, France
}

\begin{abstract}
We address the problem of computing the exact characteristics of any subsegment of a digital straight line with known characteristics. We present a new algorithm that solves this problem, whose correctness is proved. Its principle is to climb the Stern-Brocot tree of fraction in a bottom-up way. Its worst-time complexity is proportionnal to the difference of depth of the slope of the input line and the slope of the output segment. It is thus logarithmic in the coefficients of the input slope. We have tested the effectiveness of this algorithm by computing a multiscale representation of a digital shape, based only on a digital straight segment decomposition of its boundary.
\end{abstract}

Keywords: standard lines, digital straight segment recognition, SternBrocot tree.

\section{Introduction}

Digital Straight Lines (DSL) and Digital Straight Segments (DSS) are useful to describe the geometry of a digital shape (coding, discrete geometric estimators, feature detection) and this explains why they have been so deeply studied (see the survey [9] or [8]). When a straight line is digitized on a grid, we obtain a sequence of grid points defining a digital straight line segment. Methods of recognizing digital straight segments are known since long. In one of the first methods, Freeman [7] suggested to analyze the regularity in the pattern of the directions in the chain code [6] of a digital curve. Anderson and Kim [1] have presented a deep analysis of the properties of DSS and suggested a different algorithm based on calculating the convex hull of the points of digital curves to be analyzed. In [10], Kovalevsky presented a new classification of digital curves into boundary curves and visual curves. Boundary curves and lines are a useful mean for fast drawing of regions defined by their boundaries. Modern DSS recognition algorithms achieves a computational complexity of $O(n)$, if $n$ is the number of input points and with a computing model where arithmetic operations takes $O(1)$ time. Interestingly, this complexity is obtained by algorithms based on arithmetic properties [1211], combinatorial properties [16], or dual-space construction 5]. These algorithms 
are optimal, when no further information is known. However, in some case, we may already know that a set of points is included in some DSL of known characteristics. This happens for instance when computing the multiresolution of a digital object, since analytic formulas give the multiresolution of DSL. We assume that the digital shape was previously polygonalized as a sequence of $M$ $D S S$, for instance by a simple greedy $D S S$ segmentation algorithm or with the Minimum Perimeter Polygon [13. We then calculate the characteristics of each $D S S$ when changing the resolution of the grid (see 14 for more details about the covering of a DSL). In this case, a faster computation of the DSS parameters is possible.

Many works deal with the relations between irreducible rational fractions and digital lines (see 4 for characterization with Farey series, and 18 for a link with decomposition into continuous fractions). In [2, Debled and Réveillès first introduced the link between the Stern-Brocot tree and the recognition of digital line. Recognizing a piece of digital line is like going down the Stern-Brocot tree up to the directional vector of the line. To sum up, the classical online DSS recognition algorithm DR95 [2] (also reported in 8]) updates the DSS slope when adding a point that is just exterior to the current line (weak exterior points). In [17, De Vieilleville and Lachaud have revisited a classical arithmetically-based DSS recognition algorithm with new parameters related to a combinatorial representation of DSS. New analytic relations have been established and the relation with the Stern-Brocot tree has been made explicit.

The main contribution of this paper is to present a fast algorithm which computes the exact (minimal) characteristics of a DSS that is some subset of a DSL of known characteristics (see [15 for more details about minimal characteristics). More precisely, the input DSL, say $D$, is given as the continued fraction of its slope. The DSS is specified by the positions of its two endpoints $A$ and $B$. Furthermore, the two lower leaning points of $D$ surrounding $A$ and $B$ are given as input. This new algorithm, called ReversedSmartDS\$1, determines the characteristics of the DSS by moving in a bottom-up way along the Stern-Brocot tree, a famous tree structure that represents positive fractions. We prove the correctness of this algorithm in Proposition 1. We further show in Proposition 2 that its worst-case computational complexity is $\Theta\left(k-k^{\prime}\right)$, where $\left[u_{0} ; u_{1}, \ldots, u_{k}\right]$ is the continued fraction of the slope of the input DSL and $\left[u_{0} ; u_{1}, \ldots, u_{k^{\prime}}\right]$ is the continued fraction of the slope of the output DSS. This result assumes a computing model where standard arithmetic operations are in $O(1)$, which is a reasonnable assumption when analyzing digital shapes.

This complexity is first to compare with the SmartDSS algorithm [14, whose worst-case computational complexity is $\Theta\left(\sum_{i=0}^{k^{\prime}} u_{i} * \delta\right)$, where $\delta$ is the number of patterns of the output DSS. The average of this sum for all fractions $\frac{a}{b}$ with $a+b=n$ is experimentally lower than $\log ^{2} n$, and this sum is upper bounded by $n$ and lower bounded by 1 . This complexity is secondly to compare with the complexities of classical DSS recognition algorithms (e.g., DR95 [3], see

${ }^{1}$ This name is in opposition to the SmartDSS algorithm 14, because it moves along the Stern-Brocot in a reversed direction. 
also [9]), whose complexities are at best $\Theta(n)$. The ReversedSmartDSS algorithm was implemented and tested and various experimental results show that this algorithm performs better than the SmartDSS algorithm and the classical DSS recognition algorithms (see Table 1).

Table 1. Computation times of the $(h, v)$-covering of various digital shapes with our proposed approach. The digital shapes are: a circle of radius 2000; a flower with 5 petals, mean radius 5000 and variability of radius 7000; a polygon with 8 sides and radius 2000 . The symbol \# stands for "number of".

\begin{tabular}{|c|c|c|c|c|c|c|c|c|c|}
\hline Shape & \multicolumn{3}{|c|}{ Flower } & \multicolumn{3}{c|}{ Circle } & \multicolumn{3}{c|}{ Polygon } \\
\hline \hline \# points & \multicolumn{3}{|c|}{67494} & \multicolumn{3}{c|}{16004} & \multicolumn{3}{c|}{15356} \\
\hline \# segments & \multicolumn{3}{|c|}{1991} & \multicolumn{3}{c|}{574} & \multicolumn{3}{|c|}{44} \\
\hline$h, v$ & 2 & 4 & 10 & 2 & 4 & 10 & 2 & 4 & 10 \\
\hline \# points $(h, v)$ & 33744 & 16870 & 6750 & 8000 & 4000 & 1600 & 7676 & 3840 & 1532 \\
\hline \hline \multicolumn{8}{|c|}{ Smart DSS } \\
\hline \# points tested & 19352 & 11254 & 4367 & 5413 & 2977 & 1019 & 782 & 667 & 527 \\
\hline timings (ms) & 3.1286 & 2.6446 & 2.2914 & 0.997 & 0.8902 & 0.7618 & 0.1258 & 0.1142 & 0.0946 \\
\hline \hline \multicolumn{8}{|c|}{ Reversed Smart DSS } \\
\hline timings $(\mathrm{ms})$ & 2.364 & 2.103 & 2.078 & 0.758 & 0.702 & 0.625 & 0.104 & 0.097 & 0.084 \\
\hline
\end{tabular}

In Section 2 we describe this new algorithm. We show the correctness of this new algorithm in Section 3, as well as its computational complexity. The last section concludes and presents future works.

\section{A Coarsening Algorithm for Computing the Characteristics of a Subsegment Included in a Known DSL}

We recall first that a standard digital straight line $D$ in the fourth quadrant is some subset of the digital plane $\left\{(x, y) \in \mathbb{Z}^{2}, \nu \leq \alpha x+\beta y<\nu+\alpha+\beta\right\}$, where $(\alpha, \beta, \nu) \in \mathbb{Z}^{+} \times \mathbb{Z}^{+} \times \mathbb{Z}$ form the characteristics of $D$. The fraction $\frac{\alpha}{\beta}$ is the slope of the DSL. Any 4-connected piece of a DSL is a digital straight segment (DSS). The characteristics of a DSS are the characteristics of the "simplest" DSL covering it (the one with the smallest possible $\alpha$ ). Lastly, a pattern (resp. reversed pattern) is the Freeman chaincode joining two consecutive lower leaning points (resp. upper leaning points). Patterns are a combinatorial characterization of DSL.

In the next section, we describe our new algorithm for determining the characteristics of any subsegment $S$ of a digital straight line $D$ in the fourth quadrant, whose characteristics $(\alpha, \beta, \nu)$ are known. We make use of the property that the slope of $S$ is either $\frac{\alpha}{\beta}$ or any one of the ancestors of $\frac{\alpha}{\beta}$ in the Stern-Brocot tree ([15], see also Proposition 3 of [14]). The principle of our new algorithm is to follow a bottom-up way in the Stern-Brocot tree.

\subsection{Overview of the Algorithm}

Algorithm 1 is the general algorithm for computing the exact characteristics of $S$ knowing the characteristics $(\alpha, \beta, \mu)$ of its covering line $D$. This algorithm 


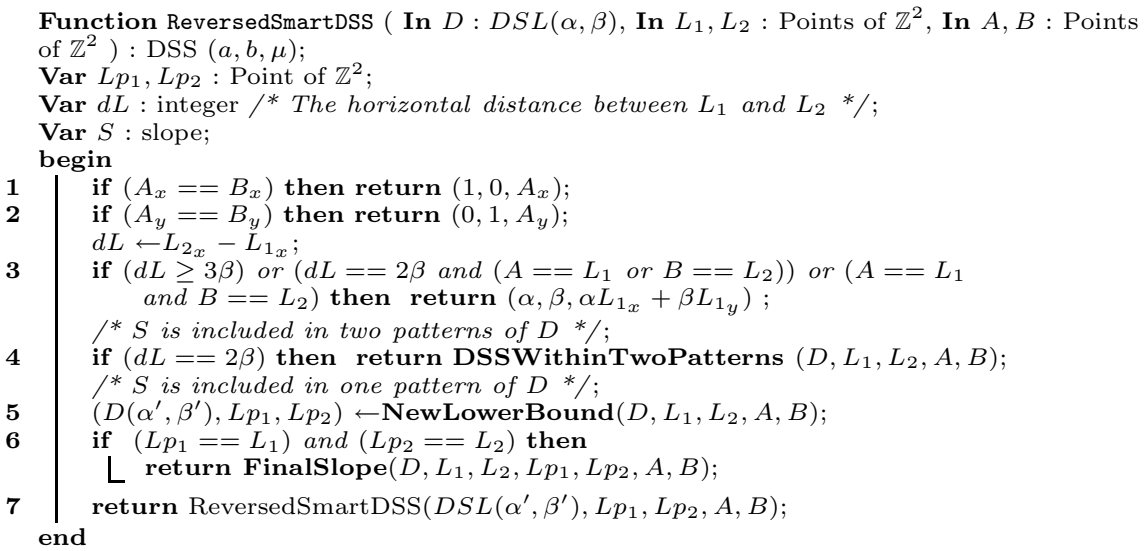

Algorithm 1. Main algorithm. Computes the characteristics $(a, b, \mu)$ of a DSS $S$ that is some subset of a DSL $D$ of slope $\frac{\alpha}{\beta}$ and lower leaning points $L_{1}$ and $L_{2}$ (the ones surrounding the segment $A B$ ). The DSS is defined by a starting point $A$ and an ending point $B(A, B \in D)$.

thus computes the simplest DSL covering $S$. The segment $S$ is defined by its two endpoints $A$ and $B$. Lastly, we give also as input the two lower leaning points of $D$ which surround $A$ and $B$. Note that these input data are all known if the DSL $D$ was recognized by a classical recognition algorithm (e.g., DR95 3]).

If $A$ and $B$ have the same abscissa (or same ordinate), then this algorithm stops and return $\left(1,0, A_{x}\right)$ (or $\left(0,1, A_{y}\right)$ ), which are the obvious results. Otherwise, this algorithm then checks the horizontal distance between $L_{1}$ and $L_{2}$ to measure the number of patterns covering the segment. Should this distance be: (1) three times greater than $\beta,(2)$ equal to $2 \beta, A$ and $L_{1}$ are superposed, or $B$ and $L_{2}$ are superposed, or (3) $A$ and $L_{1}$ are superposed, and $B$ and $L_{2}$ are superposed, then the algorithm stops and returns $(\alpha, \beta)$ (line 3 ). Indeed, in these case, the DSS contains at least one pattern, so the characteristics follow.

Otherwise, if this horizontal distance is equal to $2 \beta-S$ is included in two patterns of $D$ - then the characteristics are computed by the function DSSWithinTwoPatterns (line 4), described by Algorithm 2 .

\section{2 $S$ Is Included in Two Patterns of $D$}

The function DSSWithinTwoPatterns (Algorithm2) is really the core of this DSS recognition method. In its loop, three different patterns are tested progressively, so as to find the first that has exactly the sought slope. It is easy to see that, since the segment is included in two patterns, the middle lower leaning point $L_{m}$ is the lowest point of the segment. Therefore, if the slope of the segment is defined by a pattern (i.e. defined by two lower leaning point), then one of the extremities of the pattern is this point $L_{m}$. We thus test in sequence the possible patterns to the left and to the right of $L_{m}$. However, the slope of a DSS may 
also be defined by a reversed pattern (i.e. defined by two upper leaning points). We thus test also in sequence the possible reversed patterns.

The progressive computation of these three sequences of patterns (left pattern, right pattern, reversed pattern) is done in a parallel manner. More precisely, they are run consecutively one step at each time:

- line 4 with a call to LowerSlope(..., true) computes the next left patterns and stops when the lower leaning point $L_{1}$ overtakes or reaches $A$,

- line 5 with a call to LowerSlope(..., false) computes the next right patterns and stops when the lower leaning point $L_{2}$ overtakes or reaches $B$,

- line 6 with a call to UpperSlope computes the next reversed pattern and stops when the upper point $R U$ overtakes towards the left or reaches $B$ and the upper point $L U$ overtakes towards the right or reaches $A$.

The conditions at lines [3, 7 and 8 corresponds to three possible cases where respectively no, one, or two pattern computation(s) is/are stopped. At each iteration, the pattern, reversed or not, with deepest slope is the candidate solution. Various tests of the positions of the current leaning points with respect to the segment $A B$ allow to determine if this candidate is valid. If it is true, the function exits and returns the characteristics of the elected pattern. If it is false, the function loops and computes the new three patterns. This function loops at most $k$ times, where $k$ is the depth of the input slope.

Algorithm 3 computes in $O(1)$ the characteristics of the lower bound in the left (or right according to the boolean variable $L$ eft) of $L_{m}$. In this algorithm, we fix $L_{m}$, move $L_{1}$ right towards $A$ (or $L_{2}$ left towards $B$ in the other case) and calculate the value of the new slope between the new $L_{1}$ and $L_{m}$ (or $L_{m}$ and the new $L_{2}$ ).

Algorithm 4 determines in $O(1)$ the positions of the new upper leaning points $L U$ and $R U$, according to the parity of slope (line 133) (see [17] for more details about the parity of the slope). We have moved $L U$ and $R U$ either towards the left in the odd case or towards the right in the even case. But before moving, we must calculate the number of subpatterns, that is covering the point $A$ in the even case (line 2) or $B$ in the odd case (line 4).

Example. Let us look at a run of Algorithm 1 for the DSL $D$ of slope 13/18= $[0,1,2,1,1,2]$ (Fig. 1), for the subsegment $A B$. Here the segment $S$ is included in two patterns 13/18. Since the condition on line 3 of Algorithm 1 is fulfilled, we call DSSWithinTwoPatterns (Algorithm 2) to compute the characteristics $(a, b, \mu)$ of $S$.

For the first step, left pattern has slope 3/4 (call LowerSlope, see Fig. 2, a), right pattern has slope 5/7 (call LowerSlope, see Fig. 2. c), and reversed pattern has slope 5/7 (call UpperSlope, see Fig. 2,b). As Algorithm4 is stopped, we thus compute the deepest slope of $3 / 4,5 / 7$ and $5 / 7$, which is $5 / 7$. Since the deepest slope coincides with the slope returned by the stopped algorithm, Algorithm 2 stops and returns this slope (final result $(5,7,6)$ ).

Let us now give some explanations of (Fig. 2,a). In the first step, we fix $L_{11}$ at $L_{1}$ and $L_{22}$ at $L_{m}$, and we compute the previous slope $P S=5 / 7$ of $S=13 / 18$. Since the parity of $S$ is odd, then we calculate the number $k$ of subpatterns 
Function DSSWithinTwoPatterns( In $D$ : DSL $(\alpha, \beta)$, In $L_{1}, L_{2}$ : Lower bounds of $D$, In $A, B$ : Point of $\left.\mathbb{Z}^{2}\right)$ : DSS $(a, b, \mu)$;

Var $U_{1}, U_{2}, L_{m}$ : Point of $\mathbb{Z}^{2} ; \mathrm{L}, \mathrm{R}, \mathrm{U}$ : boolean;

Var $S_{1}, S_{2}, S_{3}, D S$ : slope;

Var $D_{L}, D_{R}, D_{U}:$ DSL $(\alpha, \beta), \mathrm{CF}$ : Continued Fraction of $D$;

begin \begin{tabular}{l|l} 
& $L \leftarrow$ true, $R \leftarrow$ true, $U \leftarrow$ true, $\mathrm{i} \leftarrow 0 ;$ \\
$\mathbf{2}$ & $L_{m} \leftarrow$ MiddleLowerBound $\left(L_{1}, D\right), C F \leftarrow$ ContinuedFraction $(D) ;$ \\
& $U_{1} \leftarrow$ FirstUpperBound $\left(D, L_{1}, L_{2}\right), U_{2} \leftarrow \operatorname{SecondUpperBound}\left(U_{1}, D\right) ;$ \\
& while $i<|C F|$ do
\end{tabular}

3

if $(L$ and $R$ and $U)$ then

$S_{1} \leftarrow$ LowerSlope $\left(D_{L}, L_{1}, L_{m}, L_{2}, A\right.$, true $) /^{*}$ Left Lower Slope */;

$S_{2} \leftarrow$ LowerSlope $\left(D_{R}, L_{1}, L_{m}, L_{2}, B\right.$, false $) /{ }^{*}$ Right Lower Slope */;

$S_{3} \leftarrow$ UpperSlope $\left(D_{U}, U_{1}, U_{2}, A, B\right) /^{*}$ Upper Slope */;

if $\left(L_{1}>=A\right.$ or $L_{2}<=B$ or $\left(U_{1}>=A\right.$ and $\left.\left.U_{2}<=B\right)\right)$ then

$\mathrm{L} D S \leftarrow$ DeepestSlope $\left(S_{1}, S_{2}, S_{3}\right) /{ }^{*} D S$ The deepest slope */;

if $L_{1}>=A$ then $L \leftarrow$ false;

if $L_{2}<=B$ then $R \leftarrow$ false;

if $\left(U_{1}>=A\right.$ and $\left.U_{2}<=B\right)$ then $U \leftarrow$ false;

if $\left(L_{1}>=A\right.$ and $\left.D S==S_{1}\right)$ or $\left(L_{2}<=B\right.$ and $\left.D S==S_{2}\right)$ or

$\left(U_{1}>=A\right.$ and $U_{2}<=B$ and $\left.D S==S_{3}\right)$ then break;

else if $((L$ and $R)$ or $(L$ and $U)$ or $(R$ and $U))$ then

$S_{1} \leftarrow(R$ and $U)$ ? LowerSlope $\left(D_{R}, L_{1}, L_{m}, L_{2}, B\right.$, false $)$

: LowerSlope $\left(D_{L}, L_{1}, L_{m}, L_{2}, A\right.$, true $)$;

$S_{2} \leftarrow(L$ and $R)$ ? LowerSlope $\left(D_{R}, L_{1}, L_{m}, L_{2}, B\right.$, false $)$

: UpperSlope $\left(D_{U}, U_{1}, U_{2}, A, B\right)$;

if $\left(\left(((L\right.\right.$ and $U)$ or $(L$ and $R))$ and $\left.\left(L_{1}>=A\right)\right)$ or $(((R$ and $U)$ or

$(L$ and $R))$ and $\left.\left(L_{2}<=B\right)\right)$ or $(((R$ and $U)$ or $(L$ and $U))$ and

$\left(U_{1}>=A\right.$ and $\left.\left.\left.U_{2}<=B\right)\right)\right)$ then $D S \leftarrow$ DeepestSlope $\left(S_{1}, S_{2}\right)$;

if $\left(\left((R\right.\right.$ and $U)$ and $\left(\left(L_{2}<=B\right.\right.$ and $\left.D S==S_{1}\right)$ or $\left(U_{1}>=A\right.$ and

$U_{2}<=B$ and $\left.\left.\left.D S==S_{2}\right)\right)\right)$ or $\left((L\right.$ and $U)$ and $\left(\left(L_{1}>=A\right.\right.$ and

$\left.D S==S_{1}\right)$ or $\left(U_{1}>=A\right.$ and $U_{2}<=B$ and $\left.\left.\left.D S==S_{2}\right)\right)\right)$ or $((L$

and $R)$ and $\left(\left(L_{1}>=A\right.\right.$ and $\left.D S==S_{1}\right)$ or $\left(L_{2}<=B\right.$ and

$\left.\left.\left.D S==S_{2}\right)\right)\right)$ ) then break;

if $\left(((L\right.$ and $U)$ or $(L$ and $R))$ and $\left.\left(L_{1}>=A\right)\right)$ then $L \leftarrow$ false;

if $\left(((R\right.$ and $U)$ or $(L$ and $R))$ and $\left.\left(L_{2}<=B\right)\right)$ then $R \leftarrow$ false;

if $\left(((R\right.$ and $U)$ or $(L$ and $U))$ and $\left(U_{1}>=A\right.$ and $\left.\left.U_{2}<=B\right)\right)$ then

$\llcorner U \leftarrow$ false;

else

if $L$ then $D S \leftarrow$ LowerSlope $\left(D_{L}, L_{1}, L_{m}, L_{2}, A\right.$, true $)$;

else if $R$ then $D S \leftarrow$ LowerSlope $\left(D_{R}, L_{1}, L_{m}, L_{2}, B\right.$, false);

else $D S \leftarrow \operatorname{UpperSlope}\left(D_{U}, U_{1}, U_{2}, A, B\right)$;

if $\left(\left(L\right.\right.$ and $\left.L_{1}>=A\right)$ or $\left(R\right.$ and $\left.L_{2}<=B\right)$ or $\left(U\right.$ and $U_{1}>=A$ and $\left.\left.U_{2}<=B\right)\right)$ then break;

$a \leftarrow D S_{x}, b \leftarrow D S_{y}, \mu \leftarrow a L_{m_{x}}+b L_{m_{y}}$

return $(a, b, \mu)$;

end

Algorithm 2. Computes the characteristics $(a, b, \mu)$ of a DSS $S$ that is some subset of a DSL $D(\alpha, \beta)$ repeated twice 
Function LowerSlope( In $D$ : DSL $(\alpha, \beta)$, InOut $L_{1}, L_{m}, L_{2}$ : Lower bounds of $D$, In $X(A$ or $B)$ : Point of $\mathbb{Z}^{2}$, In Left : Boolean): DSS $(a, b)$;

Var $P, L_{11}, L_{22}$ : Points of $\mathbb{Z}^{2} /{ }^{*} L_{11}$ and $L_{22}$ two lower leaning points */;

Var $P S$ : slope;

Var $k$ : integer;

Var parity : boolean;

begin

$\left(P, L_{11}, L_{22}\right) \leftarrow$ Left ? $\left(L_{1}, L_{1}, L_{m}\right):\left(L_{2}, L_{m}, L_{2}\right)$

parity $\leftarrow$ Parity $(D)$;

$P S \leftarrow$ PreviousSlope $(D)$;

if (parity is odd) then

$k \leftarrow$ NumberOfCoveringSubPatterns $\left(L_{11}, X, P S\right.$, true, false $)$;

$P \leftarrow L_{11}-k\left(-P S_{y}, P S_{x}\right)$

else

$k \leftarrow$ NumberOfCoveringSubPatterns $\left(L_{22}, X, P S\right.$, true, false $)$;

$P \leftarrow L_{22}-k\left(P S_{y},-P S_{x}\right)$

if Left then $L_{11}=P$;

else $L_{22}=P$;

$(a, b) \leftarrow\left(\left|P_{y}-L_{m_{y}}\right|,\left|L_{m_{x}}-P_{x}\right|\right) ;$

$\left(L_{1}, L_{2}\right) \leftarrow$ Left ? $\left(L_{11}, L_{2}\right):\left(L_{1}, L_{22}\right)$;

end

return $(a, b)$;

Algorithm 3. Computes in $O(1)$ the Lower (Left or Right) characteristics $(a, b)$ of a DSS that is some subset of a DSL $D$, given a starting point $A$ and an ending point $L_{m}$ (Left part) or given a starting point $L_{m}$ and an ending point $B$ (Right part) $(A, B \in D)$ (Left pattern is $L_{1} L_{m}$ and Right pattern is $L_{m} L_{2}$ )

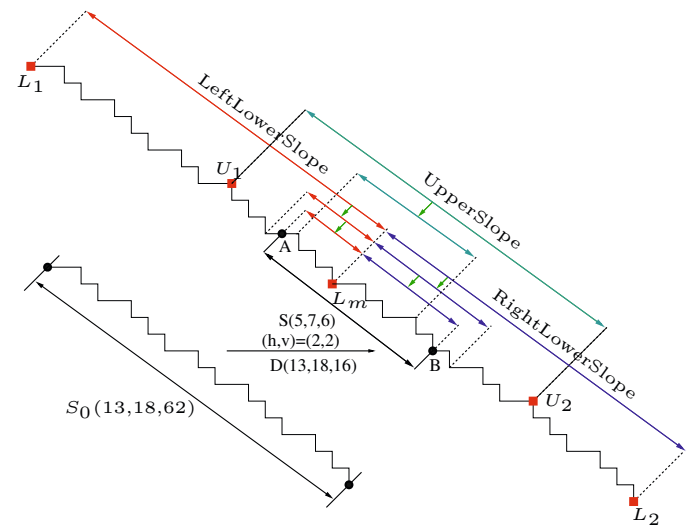

Fig. 1. A DSL $D(13,18,16)$ with two patterns between $L_{1}$ and $L_{2}$ and an odd depth slope. $S(A B)$ is the covering of $S_{0}$ by the tiling $(h, v)=(2,2)$, and also a subset of $D(A B$ is included in two patterns of $D)$. Lower and upper leaning points are drawn as red boxes. The (red, blue or cyan) arrows represent bottom-up move along the Stern-Brocot Tree.

$5 / 7$ that is covering $A$ from $L_{11}$ to the right, such that this covering reaches or overtakes $A$. It is impossible to take $k=3$, because in this case this displacement from $L_{11}$ overtakes $L_{22}$. So we take $k=2$, and $L_{11}$ is moved of two subpatterns $5 / 7$ toward the right. We have now a new DSS $S(3,4)$ between the new $L_{11}$ and $L_{22}$. In the second step, we calculate the previous slope $P S=1 / 1$ of $S=3 / 4$. Since the parity of $S$ is even, then we calculate the number $k$ of subpatterns 
Function UpperSlope( In $D$ : DSL $(\alpha, \beta)$, In $U_{1}, U_{2}$ : Upper bound of $D$, In $A, B$ : Points of $\left.\mathbb{Z}^{2}\right): \operatorname{DSS}(a, b)$;

Var $L U, R U$ : Point of $\mathbb{Z}^{2} /{ }^{*}$ Left and Right upper leaning points */;

Var $S, P S$ : slope /* PS the previous slope of $S * /$;

Var $k$ : integer /* Number of subpatterns covering $A$ or $B * /$;

Var parity : boolean /* parity of slope continued fraction */;

begin

$L U \leftarrow U_{1}, R U \leftarrow U_{2}$

if $(L U>A$ and $R U<B)$ then

$\mathrm{L}$ return $(\alpha, \beta)$;

1

parity $\leftarrow \operatorname{Parity}(D), P S \leftarrow \operatorname{PreviousSlope}(D)$;

2 if (parity is odd) then

if $(R U>B)$ then

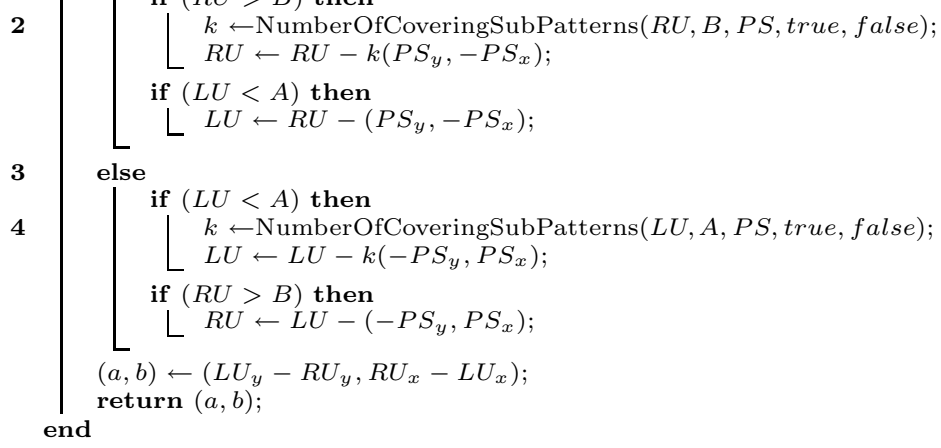

Algorithm 4. Computes in $O(1)$ the Upper characteristics $(a, b)$ of a DSS that is some subset of a DSL $D\left(U_{1}\right.$ and $U_{2}$ are two upper leaning points of $\left.D\right)$, given a starting point $A$ and an ending point $B(A, B \in D)$

$1 / 1$ that is covering $A$ from $L_{22}$ to the left, such that this covering reaches or overtakes $A$. It is impossible to take $k=4$, because in this case this displacement from $L_{22}$ overtakes $L_{11}$. So we take $k=3$, and $L_{11}$ is moved of three subpatterns $1 / 1$ toward the left and we obtain a new DSS from the new $L_{11}$ to $L_{22}$. As $L_{11}$ reach $A$, this algorithms stops and returns the left slope $1 / 1$.

\section{3 $S$ Is Included in One Pattern of $D$}

In the remaining case, $S$ is included in one pattern only. The function New LowerBounds (Algorithm 5) attempts to move $L_{1}$ toward $A$ with $k_{1}$ subpatterns and $L_{2}$ toward $B$ with $k_{2}$ subpatterns, according to the parity of the depth of the development of the slope in continued fractions (it means that Parity $(D)$ is true if $D$ has a slope with even depth, false otherwise) and to the previous convergent of the slope (line 5) (if the depth of the slope is a $k-t h$ convergent, then the previous slope is a $(k-1)-t h$ convergent). This displacement is constrained by the fact that the leaning points must still surround $A$ and $B$ (see Fig. 3. (a),(b)). In the case where $L_{1}$ and $L_{2}$ did not move, we can conclude on the characteristics with a call to FinalSlope (Algorithm 6). In this algorithm, we focus on the three cases. Firstly, if the second lower leaning point $L p_{2}$ is equal to $B$ and $L p_{1}$ moves toward $A$ with either one previous slope or previous previous slope, according to the parity of the depth of the slope. Secondly, if the first lower leaning point $L p_{1}$ is equal to $A$ and $L p_{2}$ moves towards $B$. Finally, if the lower leaning points 


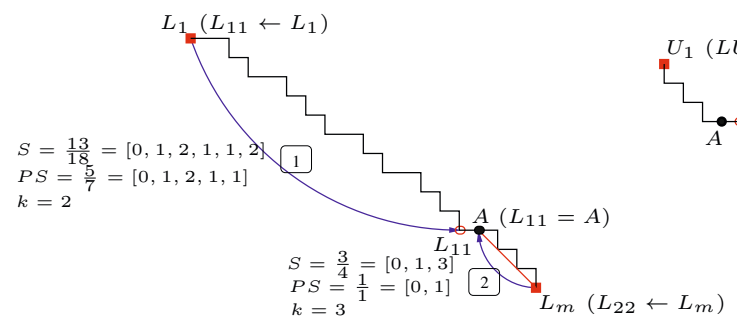

(a)

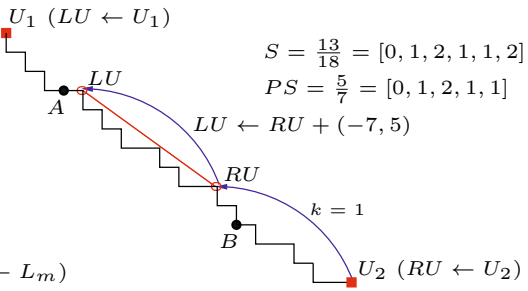

(b)

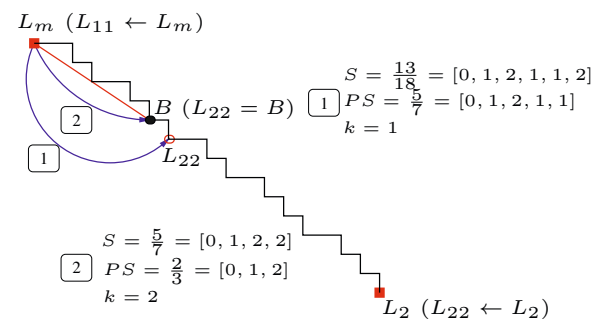

(c)

Fig. 2. The DSS $U_{1} U_{2}$ (resp. $L_{1} L_{m}$ and $L_{m} L_{2}$ ) of characteristics $(13,18,16)$, which is a subset of $L_{1} L_{2}$ of Fig. [1. The blue arrows represent the move between the upper (lower) leaning points, and $k$ is the number of subpatterns covering $B(A$ and $B)$.

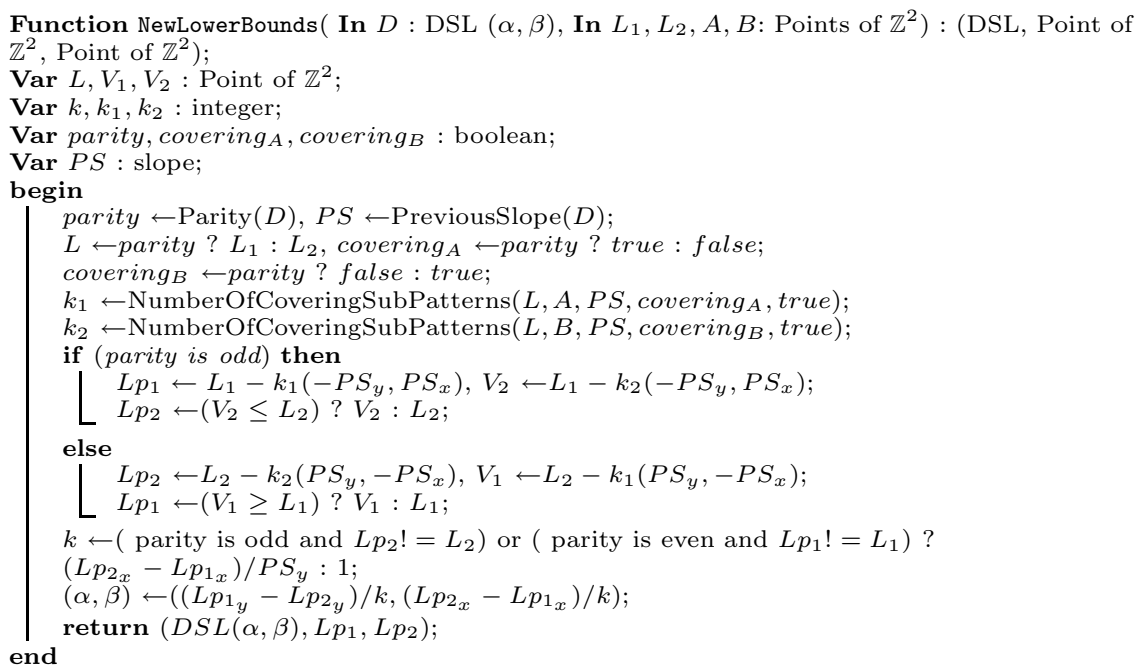

Algorithm 5. Updates in $O(1)$ the slope of the DSL $D(\alpha, \beta)$ according to the change of the two lower leaning points from $\left(L_{1}, L_{2}\right)$ to $\left(L p_{1}, L p_{2}\right)$ 


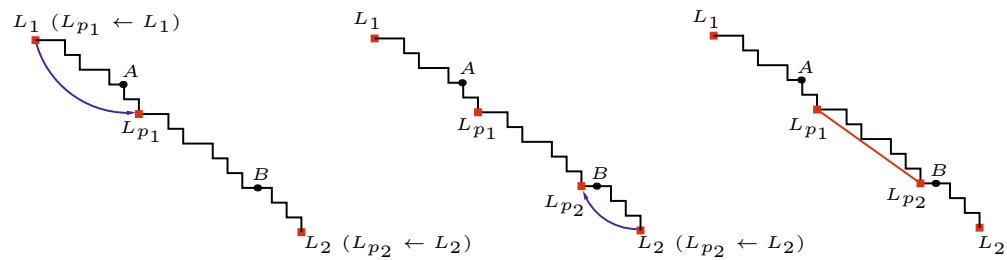
(a) $S=13 / 18, P S=5 / 7$
(b) $S=8 / 11, P S=3 / 4$
(c) $S=5 / 7$

Fig. 3. $L_{1} L_{2}$ is a DSS of characteristics $(13,18,16)$. The blue arrows represent the move of the lower leaning points. (a) $S=L_{1} L_{2}, L_{1}$ is moved of one subpatterns $5 / 7$ towards the right. (b) $S=L_{p_{1}} L_{2}, L_{2}$ is moved of one subpatterns $3 / 4$ towards the left.

(c) $L p_{1}$ and $L p_{2}$ represent the lower leaning points of $A B$.

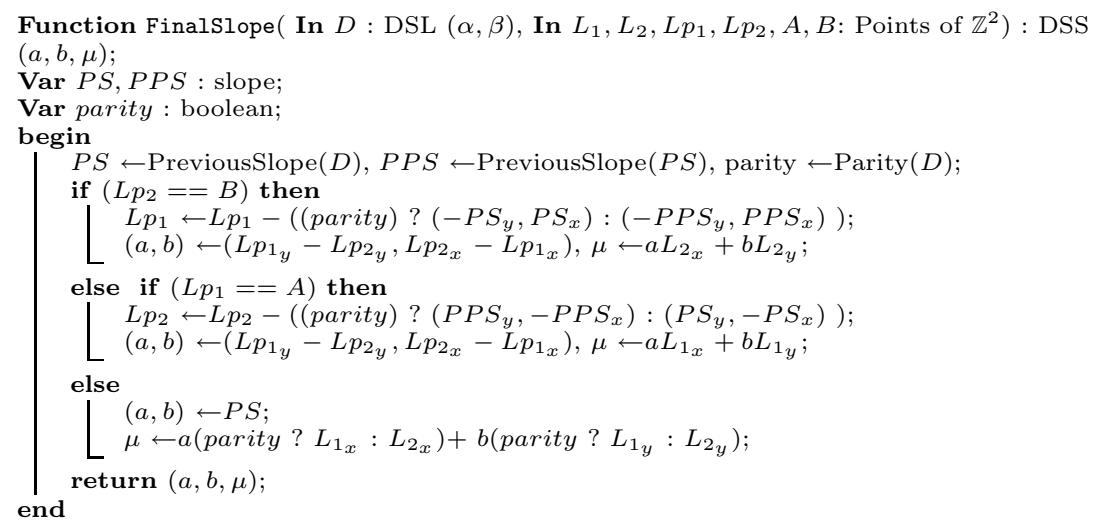

Algorithm 6. Computes in $O(1)$ the characteristics $(a, b, \mu)$ of a DSS in the case where $L_{1}=L p_{1}$ and $L_{2}==L p_{2}$

did not move. We therefore conclude that $A B$ has the slope of $L p_{1} L p_{2}$ (see Fig. 3. (c)). If at least one of the leaning points has moved, then we recursively call ReversedSmartDSS but with an input DSL with a slope depth at least one smaller than the slope depth of $D$.

\section{Correctness and Computational Complexity}

We sketch here the proof of the correctness of algorithm ReversedSmartDSS (Proposition 1). Proposition 2 establishes the time complexity of this algorithm, as a function of the depth of the slopes of the input DSL and output DSS.

Proposition 1. For any $D S L D$ such that $A, B \in D$, Algorithm 1 computes the characteristics of the segment $S=[A B]$ included in $D$.

Proof. We prove by induction on $n$ (the depth of the slope of $D$ ) that Algorithm1 computes the characteristics of the segment $S$ in the fourth quadrant. The initial steps $n=-1$ or $n=0$ are obvious since $D$ is just a vertical or horizontal 
segment and has slope $1 / 0$ or $0 / 1$. In this case, Algorithm 1 returns the correct characteristics $\left(1,0, A_{x}\right)$ or $\left(0,1, A_{y}\right)$. The induction hypothesis is that this algorithm has a correct output for all points $A, B \in D$ of slope $\left[u_{0}, u_{1}, \cdots, u_{n}\right]$. We shall prove that the output is also correct for any points $A, B$ in some DSL $D$ with a depth of its slope equal to $n+1$.

If the horizontal distance between the lower leaning points $L_{1}$ and $L_{2}$ verifies one of the three conditions of line 3 , then Algorithm 1 stops and returns the characteristics of the segment $A B$ which are trivially in this case the characteristics of $D$ itself (i.e. depth is $n+1$ ).

Otherwise, if this distance is equal to $2 \beta$, then we call Algorithm 2 There, three algorithms are run in a parallel manner: (i) LowerSlope ( . ., true) at line 4 for the pattern to the left of $L_{m}$, (ii) LowerSlope(..., false) at line 5 for the pattern to the right of $L_{m}$, and (iii) UpperSlope at line 6 for the reversed pattern containing $L_{m}$. More precisely, they are run consecutively one iteration each time. This algorithm does not depend on the induction hypothesis. It just calculates the largest pattern contained in $[A B]$ either to the left or right of the lowest point $L_{m}$, and the largest reversed pattern containing $L_{m}$. Since patterns characterize a DSS slope, the deepest slope is exactly the slope of $A B$.

The last case occurs when $S$ is included in only one pattern of $D$. We then look for a simpler DSL than $D$ that contains $A B$ by moving the lower leaning points toward $A B$ (function NewLowerBounds). The simpler DSL is one of the left or right ancestor of the slope of $D$ in the Stern-Brocot tree, according to its parity. If such a simpler DSS includes $A B$, then the lower leaning points are moved. Here, we recursively call ReversedSmartDSS but with this new input data, where the input DSL has a slope depth less or equal to $n$. The induction hypothesis applies in this case. If no simpler DSS contains $A B$, the function FinalSlope determines directly (without loop) the correct characteristics, with simple checks. We can therefore conclude in all cases.

We assume that we have stored all the convergents of the slope of $D$ before running Algorithm 1. We further assume a computing model where standard arithmetic operations takes $O(1)$. Note that the largest integer used in the presented algorithms is lower than $\alpha^{2}+\beta^{2}$, if $D$ has slope $\frac{\alpha}{\beta}$, for a frame centered on the DSS.

Proposition 2. Algorithm 1 takes $O\left(n-n^{\prime}\right)$ time complexity, where $n$ is the depth of the input DSL $D$ with slope $\frac{\alpha}{\beta}=\left[u_{0}, u_{1}, \cdots, u_{n}\right]$ and $n^{\prime}$ is the depth of the output DSS $S$ with slope $\frac{a}{b}=\left[u_{0}, u_{1}, \cdots, u_{n^{\prime}}\right]$.

Proof. Computation of Algorithm 1 on line 1, 2] and [3 is clearly $O(1)$. Otherwise, if $S$ is included in two patterns of $D$ (line 4), we then apply Algorithm 2 (detailed in the previous proposition). In the core of this algorithm, we call three algorithms where the time complexity of each algorithm is $O(1)$. Furthermore, we repeat either the same condition (line 3) until one of the three algorithms is stopped, line 7 if one is stopped, or line 8 if two are stopped. Since the stopping occurs when the output slope is reached, the number of steps of 
Algorithm 2 is the difference between the depth slope of the input DSL and the one of the output DSS, that is $O\left(n-n^{\prime}\right)$.

Otherwise, it means that $S$ is included in one pattern of $D$. In this case, we compute in $O(1)$ the positions of the new two lower leaning points $L_{1}$ and $L_{2}$ of $D$ by calling NewLowerBounds (line 5). When the lower leaning points do not move, then the function FinalSlope determines the correct characteristics in $O(1)$. Otherwise, we recursively call ReversedSmartDSS but with an input DSL with a slope depth less or equal to $n-1$. The preceding arguments recursively hold. With this observation, the worst-case computational complexity of Algorithm 1 is clearly $O\left(n-n^{\prime}\right)$.

Lamé's theorem implies that Algorithm 1 takes at most $O(\log (\max (\alpha, \beta)))$ time.

Timing measures. Execution times were measured for some contours (Table1). These times were obtained on a $2.10 \mathrm{GHz}$ Intel Core 2 Duo. The listed numbers include the computation time for subsampling contours and the associated number of tested points in the SmartDSS algorithm [14] and the computation of the characteristics of each segment in the ReversedSmartDSS algorithm.

\section{Conclusion}

We have presented a novel fast DSS recognition algorithm with guaranteed logarithmic complexity, in the special case where a DSL container is known. The algorithm principle is to move in a bottom-up way along the Stern Brocot Tree, starting from the initial known DSL slope. Finally, we have used this algorithm to efficiently compute the exact multiscale covering of a digital contour (Table 1). Our algorithms are sensitive to the depth of the input DSL and output DSS, and are clearly sublinear.

\section{References}

1. Anderson, T.A., Kim, C.E.: Representation of digital line segments and their preimages. Computer Vision, Graphics, and Image Processing 30(3), 279-288 (1985)

2. Debled-Rennesson, I.: Etude et reconnaissance des droites et plans discrets. Ph.D. thesis, Université Louis Pasteur, Strasbourg (1995)

3. Debled-Rennesson, I., Reveillès, J.P.: A linear algorithm for segmentation of discrete curves. International Journal of Pattern Recognition and Artificial Intelligence 9, 635-662 (1995)

4. Dorst, L., Smeulders, A.W.M.: Discrete representation of straight lines. IEEE transactions Pattern Analysis Machine Intelligence 6, 450-463 (1984)

5. Dorst, L., Smeulders, A.W.M.: Discrete straight line segments: Parameters, primitives and properties. In: Vision Geometry, Series Contemporary Mathematics, pp. 45-62. American Mathematical Society, Providence (1991)

6. Freeman, H.: On the encoding of arbitrary geometric configurations. Transactions on Electronic Computer 10(2), 260-268 (1961)

7. Freeman, H.: Computer processing of line-drawing images. ACM Comput. Surv. 6(1), 57-97 (1974) 
8. Klette, R., Rosenfeld, A.: Digital Geometry - Geometric Methods for Digital Picture Analysis. Morgan Kaufmann, San Francisco (2004)

9. Klette, R., Rosenfeld, A.: Digital straightness-a review. Discrete Applied Mathematics 139(1-3), 197-230 (2004), http://www.sciencedirect.com/science/ article/B6TYW-49YD4PJ-4/2/8a755750eee9d6517adbff2f20ee7dc2, the $2001 \mathrm{In}-$ ternational Workshop on Combinatorial Image Analysis

10. Kovalevsky, V.: Applications of digital straight segments to economical image encoding. In: Ahronovitz, E. (ed.) DGCI 1997. LNCS, vol. 1347, pp. 51-62. Springer, Heidelberg (1997)

11. Kovalevsky, V.A.: New definition and fast recognition of digital straight segments and arcs. In: International Conference on Pattern Analysis and Machine Intelligence, pp. 31-34 (1990)

12. Kovalevsky, V.A., Fuchs, S.: Theoretical and experimental analysis of the accuracy of perimeter estimates. In: Forster, W., Ruwiedel, S. (eds.) Robust Computer Vision, pp. 218-242 (1992)

13. Montanari, U.: A note on minimal length polygonal approximation to a digitized contour. Communications of the ACM 13(1), 41-47 (1970)

14. Said, M., Lachaud, J.-O., Feschet, F.: Multiscale Discrete Geometry. In: Brlek, S., Reutenauer, C., Provençal, X. (eds.) DGCI 2009. LNCS, vol. 5810, pp. 118-131. Springer, Heidelberg (2009), http://hal . archives-ouvertes.fr/hal-00413681/en/

15. Sivignon, I., Dupont, F., Chassery, J.M.: Digital intersections: minimal carrier, connectivity, and periodicity properties. Graphical Models 66(4), 226-244 (2004)

16. Troesch, A.: Interprétation géométrique de l'algorithme d'euclide et reconnaissance de segments. Theor. Comput. Sci. 115(2), 291-319 (1993)

17. de Vieilleville, F., Lachaud, J.O.: Revisiting digital straight segment recognition. In: Kuba, A., Palágyi, K., Nyúl, L.G. (eds.) DGCI 2006. LNCS, vol. 4245, pp. 355366. Springer, Heidelberg (2006), http://www.lama.univ-savoie.fr/ lachaud/ Publications/LACHAUD-JO/publications.html\#deVieilleville06a

18. Yaacoub, J.: Enveloppes convexes de réseaux et applications au traitement d'images. Ph.D. thesis, Université Louis Pasteur, Strasbourg (1997), http://lsiit.u-strasbg.fr/Publications/1997/Yaa97 